\title{
The semantic basis of taste-shape associations
}

Carlos Velasco, Andy T Woods, Lawrence E Marks, Adrian David Cheok, Charles Spence

Previous research shows that people systematically match tastes with shapes. Here, we assess the extent to which matched taste and shape stimuli share a common semantic space and whether semantically congruent versus incongruent taste/shape associations can influence the speed with which people respond to both shapes and taste words. In Experiment 1, semantic differentiation was used to assess the semantic space of both taste words and shapes. The results suggest a common semantic space containing two principal components (seemingly, intensity and hedonics) and two principal clusters, one including round shapes and the taste word "sweet", and the other including angular shapes and the taste words "salty", "sour", and "bitter". The former cluster appears more positively-valenced whilst less potent than the latter. In Experiment 2, two speeded classification tasks assessed whether congruent versus incongruent mappings of stimuli and responses (e.g., sweet with round versus sweet with angular) would influence the speed of participants' responding, to both shapes and taste words. The results revealed an overall effect of congruence with congruent trials yielding faster responses than their incongruent counterparts. These results are consistent with previous evidence suggesting a close relation (or crossmodal correspondence) between tastes and shape curvature that may derive from common semantic coding, perhaps along the intensity and hedonic dimensions. 
2 RUNNING HEAD: TASTE-SHAPE SEMANTICS

3

4

\section{THE SEMANTIC BASIS OF TASTE-SHAPE ASSOCIATIONS}

Carlos Velasco ${ }^{1,2}$, Andy T. Woods ${ }^{1,3}$, Lawrence E. Marks ${ }^{4,5}$, Adrian David Cheok ${ }^{2,6}, \&$ Charles Spence ${ }^{1}$

1. Crossmodal Research Laboratory, Department of Experimental Psychology, University of Oxford, Oxford, UK

2. Imagineering Institute, Iskandar, Malaysia

3. Xperiment, Surrey, UK.

4. Sensory Information Processing, John B. Pierce Laboratory, New Haven, Connecticut, USA

5. School of Public Health and Department of Psychology, Yale University, New Haven, Connecticut, USA

6. School of Mathematics, Engineering, and Computer Science, City University, London, United Kingdom

DATE: DECEMBER, 2015

\section{RESUBMITTED TO: PEERJ}

Correspondence concerning this article should be addressed to Dr. Carlos Velasco, Imagineering Institute, Anchor 5, Mall of Medini, No.4 Lebuh Medini Utara, Medini, Iskandar, Nusajaya, 79250, Johor, Malaysia. Tel: +60 $07 \quad 5096568$ Fax: +60 $07 \quad 509$ 6713. E-mail: carlos@imagineeringinstitute.org 
31 Previous research shows that people systematically match tastes with shapes. Here, we assess the

32 extent to which matched taste and shape stimuli share a common semantic space and whether

33 semantically congruent versus incongruent taste/shape associations can influence the speed with

34 which people respond to both shapes and taste words. In Experiment 1, semantic differentiation

35 was used to assess the semantic space of both taste words and shapes. The results suggest a 36 common semantic space containing two principal components (seemingly, intensity and hedonics)

37 and two principal clusters, one including round shapes and the taste word "sweet", and the other 38 including angular shapes and the taste words "salty", "sour", and "bitter". The former cluster

39 appears more positively-valenced whilst less potent than the latter. In Experiment 2, two speeded 40 classification tasks assessed whether congruent versus incongruent mappings of stimuli and 41 responses (e.g., sweet with round versus sweet with angular) would influence the speed of 42 participants' responding, to both shapes and taste words. The results revealed an overall effect of 43 congruence with congruent trials yielding faster responses than their incongruent counterparts.

44 These results are consistent with previous evidence suggesting a close relation (or crossmodal 45 correspondence) between tastes and shape curvature that may derive from common semantic 46 coding, perhaps along the intensity and hedonic dimensions. 
52

"She laughed, a laugh sweeter than honey, with a sound curving and zigzagging, as if singing" - (Mo Yan, Ball-Shaped Lightning, cited by Yu, 2003, p. 190).

\section{Introduction}

Several studies show that people systematically match both basic taste words and tastants (i.e., chemicals that generate gustatory sensations) with shapes that vary in terms of their curvature (see Cytowic \& Wood, 1982; Spence \& Deroy, 2013; Spence \& Ngo, 2012, for reviews). Over the last few years, researchers, including ourselves, have studied crossmodal (taste-shape) correspondences $^{1}$, providing some hints as to their underlying mechanisms (e.g., Velasco, Woods, Deroy, \& Spence, 2015a; Velasco, Woods, Liu, \& Spence, 2016) and their effects on taste (or gustatory) information processing more generally (e.g., Gal, Wheeler, \& Shiv, 2007; Liang, Roy, Chen, \& Zhang, 2013). Notably, while the way in which people match basic tastes with shapes seems reasonably well understood, the mechanisms that underlie crossmodal correspondences, as revealed in crossmodal (taste-shape) matches and congruency effects in perceptual processing are still to be clarified. In particular, research still needs to clarify when, how, and why the mechanism(s) that underlies taste-shape correspondences may influence the processing of taste (perceptual and linguistic) and shape information.

Velasco and his colleagues have investigated how people match basic tastes and shapes. So, for example, the results of one series of four experiments revealed that people associate sweet (both when presented as a word and as a tastant) with round shapes, and bitter, salty, and sour (as words and tastants) with more angular shapes (Velasco et al., 2015a, see also Ngo et al., 2013; Velasco,

\footnotetext{
${ }^{1}$ Here, we are mainly interested in the way in which taste/shape correspondences occur in the general population. As a consequence, research on synaesthesia, a rare condition where the stimulation of one sensory modality leads to concurrent sensory experiences in the same or other modalities, falls out of the scope of the present study. It is important to mention though, that cases of taste-shape synaesthesia have been reported elsewhere (Cytowic, 1993; Cytowic \& Wood, 1982).
} 
71 Salgado-Montejo, Marmolejo-Ramos, \& Spence, 2014; Velasco, Woods, Hyndman, \& Spence,

72 2015b; Wan et al., 2014). What is more, Velasco et al. (2015a) also reported that the more the

73 participants liked the taste (but not a taste word), the rounder the shape matched to it (see also Bar

$74 \&$ Neta, 2007, on curved objects preference) and suggested a hedonic mechanism to explain the

75 crossmodal matching (see also Ghoshal, Boatwright, \& Malika, 2015). This finding was

76 subsequently replicated by Velasco et al. (2016). The latter researchers found that taste

77 concentration can also affect shape matching, with more versus less intense tastants more likely

78 matched to angular versus round shapes, respectively (see also Becker, van Rompay, Schifferstein,

$79 \&$ Galetzka, 2011). Given the focus of the present study - on the semantic basis of taste word/shape

80 correspondences, and associated congruence effects - the aforementioned findings are intriguing.

81 Nevertheless, correlations alone do not suffice to show that a hedonic mechanism underpins the

82 correspondences. Moreover, it is important to evaluate a wider range of intensities, given that the

83 authors tested just two concentrations.

84 The idea that certain crossmodal correspondences may be mediated by the affective properties of

85 the matching or mismatching stimuli is certainly not new (e.g., Kenneth, 1923; see also Marks,

86 1996, for a review). Indeed, researchers have demonstrated recently that the way in which people

87 match music and colours (Palmer, Langlois, \& Schloss, 2015; Palmer, Schloss, Xu, \& Prado-León,

88 2013) and colours and fragrances (Guerdoux, Trouillet, \& Brouillet, 2014; Schifferstein \&

89 Tanudjaja, 2004) can be mediated by emotion. In an earlier study, Collier (1996) demonstrated

90 that judgments of many sets of visual and auditory stimuli could be reduced to two dimensions,

91 identified, by the author, as valence and activity. Further, some of the visual and auditory stimuli

92 overlapped on these dimensions. Collier's generalization is relevant to taste/shape

93 correspondences, which also might overlap on the same affective dimensions. What is more, such 
94 an idea is important in the context of multisensory perception in general, given that, in addition to

95 factors such as spatiotemporal alignment and semantic congruence (Stein, 2012), crossmodal

96 correspondences can also help mediate multisensory perception (e.g., Parise, Knorre, \& Ernst,

97 2014). Indeed, crossmodal correspondences can provide relevant information to people when they

98 make inferences about the (often) noisy sensory world (Parise, 2015).

99 Importantly, earlier research also points to the notion that taste/shape correspondences may

100 influence the processing of taste-information. For instance, Liang et al. (2013) assessed the

101 influence of shapes on people's sensitivity to sweetness using near-threshold sucrose solutions. In

102 their study, people rated round shapes as more pleasant. Further, presenting a round shape rather

103 than an angular shape before tasting a sweet solution enhanced sweetness sensitivity.

104 Unfortunately, however, this study is the only of its kind, and replication is critical (the effect is

105 certainly specific and small), perhaps using everyday, suprathreshold, solutions. Moreover, there

106 is a possible confound of response bias in the study, as Liang et al. did not attempt to control for

107 the participants' response criterion (e.g., apparently they did not include any 'blank,' water trials).

108 Gal, Wheeler, and Shiv (2007), reported a study in which their participants were asked to indicate

109 which of three shapes (which could be all round or angular) had the largest surface area before

110 rating a piece of cheddar cheese. The results showed that the curvature of the shapes presented in

111 the first task influenced the perceived sharpness of the cheese, with the angular shapes leading to

112 higher sharpness ratings than the round shapes. What is more, other studies have shown that the

113 shape of a plate and food (when it is round as compared to angular) can influence participants'

114 sweetness ratings of the food (resulting in people rating the food as tasting sweeter, see Fairhurst,

115 Pritchard, Opsina, \& Deroy, 2015; Stewart \& Goss, 2013; see also Piqueras-Fiszman, Alcaide,

116 Roura, \& Spence, 2012).. 
117 Here it is worth mentioning that, in spite of their perceptual basis, similarities across the senses

118 also surface in language (e.g., see the quote at the beginning of the Introduction, Marks, 1978,

119 1996). With this in mind, we ask whether the potential hedonic- and intensity-related

120 explanations/mediations of taste/shape correspondences may extend to taste words and, if so,

121 whether they reflect: a perceptual process; a common connotative meaning (Walker, 2012; Walker,

122 Walker, \& Francis, 2013; see also Collier, 1996; Karwoski, Odbert, \& Osgood, 1942, for earlier

123 examples); or perhaps, a combination of the two (see also Walker \& Walker, in press). According

124 to the semantic coding hypothesis ( $\mathrm{SCH}$, see Martino \& Marks, 2001), high level mechanisms that

125 connect information across the senses may emerge from developmental experiences with various

126 percepts that are coded into language, and that can affect multiple levels of human information

127 processing. Consequently, crossmodal congruence effects can arise not only in the processing of

128 perceptual stimuli, but also in the processing of verbal stimuli (Martino \& Marks, 1999). While

129 Liang et al. provided some evidence that congruent shapes can influence people's detection of

130 sweet solutions presented at near threshold levels, we ask here whether the congruence of taste

131 words and shapes can affect perceptual processing. As Marks (1978) pointed out, "According to

132 the Oxford English Dictionary, 'sharp' applied first to touch, then subsequently to taste (ca. 1000),

133 visual shape (1340), and hearing" (p. 190), indicating that shape-related words have been used to

134 describe tastes for several centuries, and thereby perhaps some kind of implicit relation between

135 shape and taste quality (see also Williams, 1976; Yu, 2003).

136 Here, we describe two experiments designed to assess whether shapes and taste words share a

137 common semantic space and whether congruence between them can influence both taste words 138 and shape information processing. Experiment 1 used semantic differentiation (Osgood, Suci, \&

139 Tannenbaum, 1957) to assess whether taste words and shapes share common dimensions of 
140 connotative meaning. Experiment 2 used a speeded classification task to assess whether taste/shape

141 congruence affects the categorization of taste words and shapes. We hypothesized that taste words

142 and shapes share a common semantic space to which previously reported associations will project,

143 and that people will respond faster to the congruent versus incongruent pairings.

\section{EXPERIMENT 1}

\section{Methods and materials}

Participants. 102 participants $(\mathrm{M}$ age $=34.7$ years, $\mathrm{SD}=11.8$, age range $=19-70,51$ females $)$

148 took part in the study, online through the Adobe Flash based Xperiment software

149 (http://www.xperiment.mobi). The participants were recruited using Amazon's Mechanical Turk

150 in exchange for a payment of 1.50 USD (see Woods, Velasco, Levitan, Wan, \& Spence 2015, for

151 a methodological overview of internet-based research). All of the participants were based in the

152 USA, and all agreed to take part in the study after reading a standard consent form. The experiment

153 was reviewed and approved by the Central University Research Ethics Committee at the University

154 of Oxford (MS-IDREC-C1-2014-056).

155 Apparatus and Materials. The images of four shapes (previously used by Köhler, 1929 and

156 Ramachandran \& Hubbard, 2001), two angular and two round (see Figure 1), as well as four taste

157 words, namely bitter, sour, salty, and sweet, were the stimuli in this study. The taste words were 158 presented in font Times New Roman 80. 
162 Each stimulus was assessed using the semantic differential technique (SDT). This is a procedure

163 in which the connotative meaning of objects and concepts is measured by using rating scales with

164 polar adjectives (see the original work of Osgood, Suci, \& Tannenbaum, 1957, for details; see also

165 Albertazzi, Cana, Dadam, \& Micciolo, 2014, for a recent example). Twelve pairs of polar

166 adjectives were included, which were based on previous research using the SDT (Osgood, Suci, \&

167 Tannenbaum, 1957; Osgood, 1964). Each pair has been found to correlate with three bipolar

168 dimensions, namely, evaluation, potency, and activity. The pairs of adjectives were: (1) nice-

169 awful, (2) good-bad, (3) mild-harsh, (4) happy-sad (evaluation), (5) powerless-powerful, (6) weak-

170 strong, (7) light-heavy, (8) shallow-deep (potency), (9) slow-fast, (10) quiet-noisy, (11) passive-

171 active, and (12) dead-alive (activity). Each shape and taste stimulus was rated on a 100-point visual

172 analogue scale (VAS), unmarked except for the adjectives, located outside the poles of the scale.

173 Adjectives within the pairs 1, 3, 6, 8, 10, and 12 were reversed during in the experiment.

174 Procedure. At the beginning of the study, all participants were informed about the general aims

175 and agreed to take part after reading a standard consent form. In the instructions, the participants

176 were told that they would be presented with taste words or shapes and asked to rate them on a

177 number of different VAS scales. On each trial, one of the stimuli (shape or taste word) was

178 presented in the middle of the screen together with a VAS (see the example in Figure 2). Trials

179 were blocked by pair of adjectives (scales), and both order of trials and order of blocks were

180 randomized across participants. In each block of adjective-defined scales, participants responded

181 to the eight stimuli (four shapes and four taste words), giving rise to a total of 96 trials.

182

183

INSERT FIGURE 2 ABOUT HERE

184 
185 Analysis. A varimax-rotated principal component analysis (PCA) was used to define the principal

186 dimensions arising from the different scale ratings of tastes and shapes. In addition, a hierarchical

187 cluster analysis with Ward's method and squared Euclidean distance as the similarity measure (see

188 Kaufman \& Rousseuw, 2005, for more details) was conducted in order to assess whether the

189 different tastes and shapes would group as a function of common ratings in the scales used in

190 Experiment 1. The aforesaid analyses were performed with IBM SPSS v. 19 and the PCA

191 visualizations were created in the R' (R Core Team, 2015) \{FactoMineR\} package (see

192 http://factominer.free.fr/). The data were aggregated as a function of dimensions and clusters and

193 Wilcoxon signed-rank tests were performed in R, to assess any difference between clusters as a

194 function of dimensions. Effect sizes were calculated by means of Cliff's Delta as implemented in

195 the \{effsize\} package in R (see https://cran.r-project.org/web/packages/effsize/effsize.pdf), in

196 which 0 indicates the absence of an effect (the distributions overlap), while a value of -1 or 1

197 indicates a large effect (no overlap whatsoever; see Cliff, 1996).

199 Results and discussion

200 The principal component analysis (PCA, see Figure 3) revealed that two components had 201 eigenvalues over Kaiser's criterion of 1 and, in combination, explained $95.98 \%$ of the variance.

202 Table 1 shows the factor loadings after the varimax (orthogonal) rotation. Note that the first and 203 second components accounted for $59.09 \%$ and $36.89 \%$ of the variance, respectively. 
207 The dendrogram resulting from the hierarchical cluster analysis appears in Figure 4. Two major 208 clusters are evident (see Table 2), one grouping round shapes with the taste word "sweet", and 209 another grouping angular shapes with the taste words salty", "sour", and "bitter". These groupings

210 reflect the tendency for stimuli in each cluster to receive similar ratings on the different semantic 211 differential scales.

212

213

214

215

216

217

218

219

220

221

222

223

224

225

226

227

228

229

230

INSERT FIGURE $4 \&$ TABLE 2 ABOUT HERE

After identifying the two principal components and the two clusters, the data were aggregated as a function of dimension and cluster (Figure 5 summarizes the mean values). Note that the scores of harsh/mild and light/heavy were reversed as they correlated negatively with their respective dimensions. Wilcoxon signed-rank tests were performed in order to assess any difference between clusters on each dimension. The ratings on the first dimension of the stimuli in the second cluster were higher than those in the first cluster, $(p<.001$, Cliff's Delta $=0.96)$, whereas the ratings on the second dimension of the stimuli in the first cluster were lower than those in the second cluster, $(p<.001$, Cliff's Delta $=0.79)$. In other words, the round shapes and the taste word "sweet" were rated as more positively-valenced and less intense than the angular shapes and the taste words "salty", "sour", and "bitter".

\section{INSERT FIGURE 5 ABOUT HERE}

These results provide further support for the presence of an association between the word "sweet" and round shapes and the words "bitter", "salty", and "sour" and angular shapes (Velasco et al., 2015a,b, 2016). Moreover, the results also suggest that tastes and shapes share a semantic space, 
231 or a set of implicit meanings, which is initially characterized by two main components. Indeed, a

232 possibility is that these components reflect the two elements identified by Velasco et al. (2015a,

233 2016), namely hedonic value and intensity. Consistently, the results of Experiment 1 are in line

234 with the idea that perceptual dimensions (e.g., sweet vs. sour, and fast vs. slow) differentiate

235 between valence and arousal in specific ways (e.g., positive and negative and high and low arousal,

236 respectively, see Cavanaugh, Maclnnis, \& Weiss, 2015). Two limitations may be mentioned in

237 regard to this study. First, it is possible that blocking by pairs of adjectives may have increased

238 contrast between stimuli in each dimension. Second, the number of shapes included is very limited.

239 This said, the common semantic space between round shapes and angular shapes, as well as their

240 differences, are certainly consistent with previous research using those shapes (Holland \&

241 Wertheimer, 1964; Lindauer, 1990; Lyman, 1979).

242 Experiment 1 provided evidence in support of the idea that taste words and visual shapes share

243 dimensions of connotative meaning. Given this, Experiment 2 aimed to assess whether the

244 crossmodal correspondence between taste words and shapes would produce congruence effects

245 over-and-above those already reported with tastes per se (Liang et al., 2013), that is, by using

246 linguistic taste stimuli. For this purpose, we designed a task in which a larger sample of participants

247 (in order to compensate for potential hardware-related differences across participants and fewer

248 trials, e.g., Woods et al., 2015) were given congruent or incongruent instructions about the

249 mapping between taste words and shapes and were later asked to respond to shapes or taste words

250 with taste words and shapes, respectively. 


\section{Methods and materials}

255 Participants. 253 participants $(\mathrm{M}$ age $=34.48$ years, $\mathrm{SD}=10.90$, age range $=18-73$ years, 138

256 females) took part in the study online and received a payment of 1.80 USD. All were based in the

257 USA, and all agreed to take part in the study after reading a standard consent form.

258

259

260

261

262

263

264

265

266

267

268

269

270

271

272

273

274

275

276

Apparatus and Materials. The ten stimuli comprised four pairs of shapes (one round and one angular within each pair, $200 \times 200$ pixels each; see Figure 6A), plus two taste words "sweet" and "sour". The taste words were again presented in font Times New Roman 80.

Procedure. The participants took part in two tasks. In one of the tasks (shape response, see Figure 6A), the participants were presented the taste words "sweet" or "sour" (one at a time) and asked to respond with either an angular or round shape (i.e., pairs of shapes taken from Experiment 1, see Figure 6B). In the other task (taste response, see Figure 6C), the participants were presented the eight shape stimuli (one at a time) and were asked to respond with the taste words "sweet" or "sour". Taste/shape congruence was manipulated in both tasks. That is, each task included a block of congruent trials and a block of incongruent trials. Both task order and block order were randomized across participants. In the congruent (incongruent) block of the taste response task, the participants were asked to respond with the word sweet every time they saw a round (angular) shape and with the word sour every time they saw an angular (round) shape. In the congruent (incongruent) block of the shape response task, the participants were instructed to respond with round shapes every time they saw the word sweet (sour), and with angular shapes every time they saw the word sour (sweet). Note, however, that the possible responses were presented to the left 
277 or to the right of the target stimulus, and the participants would have to press $\mathrm{z}$ or $\mathrm{m}$, a function of 278 the position of the correct response (see below). In both tasks, the participants were instructed to 279 respond as rapidly and accurately as possible to a target stimulus (taste words or shapes) by 280 pressing the key that was associated the stimulus that matched the parings in the instruction (shapes 281 and taste words, respectively).

282 Table 3 summarizes the experimental design. Each of the tasks included eight unique trials. In the 283 shape response task, half of the trials required of the participants to respond to the word "sweet" 284 and the other half to the word "sour". Moreover, four trials included the round shapes on the right 285 and the angular on the left (two for "sweet" and two for "sour"); in the remaining four trials, the 286 positioning was reversed. In the taste response task, the participants responded to the eight shapes with words "sweet" and "sour". The right-left position of "sweet" and "sour" was thus fully 288 counterbalanced.

289

290

291

292

293

294

295

296

297

298

299
INSERT TABLE 3 ABOUT HERE

All eight unique trials were presented, once each for practice, before each block of congruent and incongruent trials in each task. Feedback came after each of the practice trials with the word "correct" or "wrong" presented for $0.5 \mathrm{~s}$. Immediately after the practice trials, the participants proceeded to the experimental trials of the block. All eight unique trials were presented twice, giving rise to a total of 16 trials per block and 64 for the whole experiment. To prevent the participants from responding simply to the right or left position as opposed to sweet/sour or angular/round, 8 trials in each block mapped to one response ("sweet"/"sour", angular/round) to the $\mathrm{z}$ key, and in the other 8 trials to the $\mathrm{m}$ key. 
300 Analyses. Both accuracy and RTs were analysed as a function of task and congruence. Accuracy

301 and RTs were analysed by means of $2 \times 2$ analysis of variance-type statistics (ATS) with the factors

302 of task and congruence. Note that the ANOVA-type statistic, a robust rank-based nonparametric

303 alternative to the classic ANOVA, is robust to both outliers and the violation of assumptions in

304 classical parametric ANOVA (see Erceg-Hurn \& Mirosevich, 2008. The analyses were performed

305 in R Statistical Software, as implemented in the $\{$ nparLD $\}$ package (Noguchi, Gel, Brunner, \&

306 Konietschke, 2012). The significant main effects and interactions were further analysed with the

307 Wilcoxon signed-rank test to which Bonferroni corrections were also applied. Effect sizes were

308 also calculated by means of Cliff's Delta.

\section{Results and discussion}

310 Accuracy. Data from those participants failing to respond accurately on more than $60 \%$ of the trials

311 were excluded from the analyses (a total of 14 participants). Whilst there was a significant main

312 effect of task, $F_{A T S}(1, \infty)=15.15, p<.001$, the effect of congruence was not significant, $F_{A T S}(1$,

$313 \infty)=0.39, p=.530$, nor was the interaction between task and congruence, $F_{A T S}(1, \infty)=1.68, p=$

314 .195. Wilcoxon signed-rank test revealed that the participants were more accurate in the task in

315 which they had to respond with taste words rather than shapes $(p=.001$, Cliff's Delta $=0.15)$.

316 Figure 7A summarizes the results.

$R T s$. Only the trials in which the participants responded correctly were included in the analyses

321 (91.6\% of 15296 trials). The ANOVA-type statistic revealed a significant effect of task, $F_{A T S}(1$, 322

$\infty)=121.31, p<.001$ and congruence, $F_{A T S}(1, \infty)=13.71, p<.001$. The interaction between task 
323 and congruence was not significant, $F_{A T S}(1, \infty)=3.38, p=0.066$. The participants responded more

324 rapidly in the task in which they responded with taste words rather than shapes $(p<.001$, Cliff's

325 Delta $=0.27)$. Moreover, participants also responded more rapidly on the congruent than the

326 incongruent trials $(p<.001$, Cliff's Delta $=0.13)$.

327 The results of Experiment 2 provide evidence for the idea that taste/shape correspondences can

328 indeed produce congruence effects even in the absence of a tastant, but just with shapes and taste

329 words. It is worth mentioning, though, that there was a difference across tasks too: That is, the

330 participants responded more accurately, and more rapidly, in the taste response task as compared

331 to the shape response task. Moreover, an overall congruence effect was observed across tasks. The

332 results of Experiment 2 extend previous studies assessing taste/shape congruence (e.g., Fairhurst

333 et al., 2015; Liang et al., 2013) to taste word/shape congruence.

336 Two experiments aimed to assess, first, whether basic taste words and shapes share a semantic

337 space - a set of implicit meanings - that may contribute to the correspondences between these

338 stimuli, and, second, whether these crossmodal correspondences can induce congruence effects

339 when linguistic stimuli rather than tastants are used. Experiment 1 revealed that taste words and

340 shapes can share a semantic space, which is mainly characterized by dimensions related to intensity

341 and hedonic value. Moreover, consistent with previous research (Spence \& Deroy, 2013, for a

342 review), specific tastes and shapes clustered, "sweet" with round shape and "bitter", "salty", and

343 "sour" with angular shape. A limitation of Experiment 1, though, is that only four shapes were

344 used. Nevertheless, the results are consistent with previous research suggesting that the shapes 
345 used are distinctively round and angular (e.g., Holland \& Wertheimer, 1964; Lindauer, 1990;

346 Lyman, 1979), that the aforesaid groups of tastes and shapes tend to be rated similarly (Velasco,

347 2015a; 2016), and that perceptual dimensions can be described along such dimensions (Collier,

348 1996; Cavanaugh, Maclnnis, \& Weiss, 2015).

349 Experiment 2 introduced a task in which people were instructed to respond either to shapes with

350 taste words or to taste words with shapes, under conditions that defined the stimulus-response

351 relations either congruently (e.g., respond round to "sweet") or incongruently (e.g., respond

352 angular to "sweet"). Both task and congruence mattered: First, the participants were more accurate

353 and faster when responding to taste words with shapes than when responding to shapes with taste

354 words. And second, the participants responded more rapidly with congruent as compared to with

355 incongruent pairings of stimuli and responses.

356 One important question, relating to Experiment 1, is whether the semantic basis of taste words and

357 shapes may also apply to tastants. Research by Velasco et al. (2015a) demonstrated that the ways

358 in which people match taste words and tastants to shapes follow similar patterns (as might be

359 expected $)^{2}$. Sweet tends to associate with round shapes whilst bitter, sour, salty, and salty associate

360 with angular shapes. One important direction for future research concerns the evaluation of the

361 semantic space of both taste words and tastants. For example, one could examine the common

362 semantic space for taste words and tastes by running either a semantic differentiation study or a

363 similarity rating experiment on a stimulus set that included both tastes and taste words. Findings

\footnotetext{
2 This is of particular relevance given the fact that previous research has documented the importance of some nonsemantic features of taste words (e.g., typeface features, see Velasco et al., 2015b) and/or implicit vocalization, articulation/kinesthesis, and/or sound imagery in conveying meaning (e.g., Ngo et al., 2013). For example, one may argue that it is not the word "sweet" but rather its sound symbolic meaning, that guides its matching to round shapes. While we cannot rule out all the specific interactions between the aforesaid variables, it is known that tastants and taste words are similarly matched to shapes varying in terms of their curvature (Velasco et al., 2015a).
} 
364 of Gallace, Boschin, and Spence (2011) with different foods, however, do suggest that foods with

365 specific taste qualities aligned in semantic differential scales (see also Ngo et al., 2013).

366 Although the present research focused on taste words and shapes, it is worth considering whether

367 the results would be similar if we had used shape words instead of shapes. Presumably, shape

368 words would operate semantically like shapes per se, at least to the extent that taste words operate

369 semantically like tastes (although in both cases there may be some interesting differences between

370 the connotative meanings of perceptual stimuli and the analogous words, as Osgood, 1960,

371 suggested with colors and color words). In some instances, words may connote 'prototypes' that

372 cannot easily be realized in particular stimuli. Such a matter may be an interesting direction for

373 future research.

374 Nearly two decades ago, Marks (1996) highlighted that "The correspondences between primary

375 perceptual meanings and secondary linguistic ones need not be perfect-language and perception

376 do not necessarily carve the world up in precisely the same way (cf. Miller \& Johnson-Laird, 1976)

377 - but the connections are nevertheless strong" (p. 49). The results of Experiment 2 extend previous

378 work on taste/shape associations and taste and shape information processing to taste words and

379 shapes (e.g., Becker et al., 2011; Gal et al., 2007). As noted before, in the English language, for

380 example, the use of shape-related words such as "sharp" to describe tastes has a long history

381 (Marks, 1978; Williams, 1976; Yu, 2003). This said, even though the effects found in Experiment

3822 were small, so too were earlier taste/shape congruence effects reported with perceptual stimuli

383 (Liang et al., 2013; note, however, that comparing effect sizes across experimental paradigms is

384 not an easy task given their different nature), and these effects prove noteworthy given the

385 seemingly unrelated nature of basic taste words and shapes. 
386 How to interpret the fact that taste word/shape correspondences product congruence effects? In

387 order to answer this question, it is important to highlight the fact that the tasks included in

388 Experiment 2 required the participants to learn specific associations (either congruent or

389 incongruent). This said, it is reasonable to assert that there is an implicit relation between specific

390 taste words and shape curvature. In other words, participants may find it easier to respond to a

391 learned stimulus mapping that has a stronger (implicit) association than to one that has a weaker

392 association, thus responding faster to the former. How is such implicit relationship built? Results

393 of Experiment 1, together with those of Velasco et al. (2015a, 2016), provide some clues in support

394 of a hedonic association, and preliminary experimental data for a sensory-discriminative

395 association (see also e.g., Marks, 1978, 2013; Parise \& Spence, 2013; Spence, 2011, for reviews

396 on possible mechanisms underlying crossmodal correspondences). These ideas are consistent with

397 an affective account of certain associations across the senses (e.g., Collier, 1996; Palmer, Schloss,

398 Xu, \& Prado-León, 2013). Given that intensity and hedonics are also influenced by other low-level

399 visual properties and shape aesthetic features (e.g., Palmer, Schloss, \& Sammartino, 2013), which

400 influence taste/shape correspondences (Salgado-Montejo et al., 2015), it should be reasonable to

401 extend the present results to other visual attributes (e.g., shape symmetry).

402 The two experiments were conducted online. Even though there is still debate as to the limitations 403 and scope of online research, a number of studies have started to suggest that, in many situations,

404 online research can mimic laboratory results (not to mention the access to larger and more varied 405 samples of participants; see Woods et al., 2015, for a review of perception research online).

406 Nonetheless, the differences in hardware, software, and contexts across online participants may

407 certainly introduce some error (though the larger samples likely compensate, see also Chetverikov 408 \& Upravitelev, in press). 
409 The results of the present study can be of interest to both researchers and practitioners in the

410 context of food and drink design, given that specific stimulus combinations may lead to subtle

411 variations in the processing of taste-related information. Indeed, even though research will be

412 undoubtedly needed, perhaps, by changing the shape of a packaging, plate, or food, it may be

413 possible to influence, for example, the expected and perceived sweetness of foods or drinks

414 without touching their actual concentration (Velasco et al., 2014).

415 To conclude, people appear to respond differently to tastes and shapes when the mappings are

416 consistent rather than inconsistent with the correspondence - an additional piece of evidence to

417 suggest that taste words and shapes share an abstract semantic network and that the existence of

418 crossmodally shared locations in semantic space ipso facto define or characterize crossmodal 419 congruence. 
424 Albertazzi L, Canal L, Dadam J, Micciolo R. 2014. The semantics of biological forms. Perception 425 43:1365-1376. DOI: $10.1068 / \mathrm{p} 7794$

426

427

428

429

430

431

432

433

434

435

436

437

438

439

Bar M, Neta M. 2006. Humans prefer curved visual objects. Psychological Science 17:645-648. DOI: $10.1111 /$ j.1467-9280.2006.01759.x.

Becker L, Van Rompay TJL, Schifferstein HNJ, Galetzka M. 2011. Tough package, strong taste: The influence of packaging design on taste impressions and product evaluations. Food Quality and Preference 22: 17-23. DOI:10.1016/j.foodqual.2010.06.007

Cavanaugh LA, MacInnis DJ, Weiss, AM. 2015. Perceptual dimensions differentiate emotions. Cognition and Emotion (ahead-of-print): 1-16. DOI: 10.1080/02699931.2015.1070119.

Chetverikov A, Upravitelev P. in press. Online versus offline: The Web as a medium for response time data collection. Behavior Research Methods. DOI: 0.3758/s13428-015-0632-x.

Cliff N. 1996. Answering ordinal questions with ordinal data using ordinal statistics. Multivariate Behavioral Research 31:331-350. DOI: 10.1207/s15327906mbr3103_4.

Collier GL. 1996. Affective synesthesia: Extracting emotion space from simple perceptual stimuli. Motivation and Emotion 20:1-32. DOI: 10.1007/BF02251005.

Cytowic, RE. 1993. The man who tasted shapes. USA: G. P. Putnam's Sons. 
440 Cytowic RE, Wood FB. 1982. Synesthesia: II. Psychophysical relations in the synesthesia of

441 geometrically shaped taste and colored hearing. Brain and Cognition 1:36-49.

442 Erceg-Hurn, DM, Mirosevich VM. 2008. Modern robust statistical methods: An easy way to 443 maximize the accuracy and power of your research. American Psychologist 63:591-601. DOI: $444 \quad$ 10.1037/0003-066X.63.7.591.

445 Fairhurst MT, Pritchard D, Ospina D, Deroy O. 2015. Bouba-Kiki in the plate: Combining 446 crossmodal correspondences to change flavour experience. Flavour 4:22. DOI: $447 \quad 10.1186 / \mathrm{s} 13411-015-0032-2$.

Gal D, Wheeler SC, Shiv B. 2007. Cross-modal influences on gustatory perception. Available at 449 SSRN: ssrn.com/abstract $=1030197$.

Gallace A, Boschin E, Spence C. 2011. On the taste of "Bouba" and "Kiki": An exploration of 451 word-food associations in neurologically normal participants. Cognitive Neuroscience 2:3446. DOI: $10.1080 / 17588928.2010 .516820$.

453

Ghoshal T, Boatwright P, Malika M. 2015. Curvature from all angles: An integrative review and 454 implications for product design. In: Batra R, Seifert C, Brei D, eds. The psychology of design: Creating consumer appeal. New York, NY: Routledge, 91-105.

Guerdoux E, Trouillet R, Brouillet D. 2014. Olfactory-visual congruence effects stable across ages: Yellow is warmer when it is pleasantly lemony. Attention, Perception, \& Psychophysics, 76:1280-1286. DOI: 0.3758/s13414-014-0703-6.

Holland MK, Wertheimer M. 1964. Some physiognomic aspects of naming, or, maluma and takete $460 \quad$ revisited. Perceptual and Motor Skills 19:111-117. DOI: 10.2466/pms.1964.19.1.111. 
461 Karwoski TF, Odbert HS, Osgood CE. 1942. Studies in synesthetic thinking: II. The role of form

462 in visual responses to music. The Journal of General Psychology 26:199-222. DOI:

463 10.1080/00221309.1942.10545166.

464

Kaufman L, Rousseeuw PJ. 2005. Finding groups in data: An introduction to cluster analysis. 465 Hoboken, NJ: John Wiley \& Sons.

Kenneth JH. 1923. Mental reactions to smell stimuli. Psychological Review 30:77-79. DOI: 10.1037/h0068405.

468

Köhler W. 1929. Gestalt psychology. New York, NY: Liveright.

469

470

471

472

473

474

475

476

477

478

479

Liang P, Roy S, Chen ML, Zhang GH. 2013. Visual influence of shapes and semantic familiarity on human sweet sensitivity. Behavioural Brain Research 253:42-47. DOI: 10.1016/j.bbr.2013.07.001.

Lindauer MS. 1990. The effects of the physiognomic stimuli taketa and maluma on the meanings of neutral stimuli. Bulletin of the Psychonomic Society 28:151-154. DOI: 10.3758/BF03333991.

Lyman B. 1979. Representation of complex emotional and abstract meanings by simple forms. Perceptual and Motor Skills 49:839-842. DOI: 10.2466/pms.1979.49.3.839.

Marks LE. 1978. The unity of the senses: Interrelations among the modalities. New York, NY: Academic Press.

Marks LE. 1996. On perceptual metaphors. Metaphor and Symbol 11:39-66. DOI:

$$
\text { 10.1207/s15327868ms1101_3. }
$$


481 Marks LE. 2013. Weak synaesthesia in perception and language. In Simner J, Hubbard E, eds. The 482 Oxford handbook of synaesthesia. Oxford, UK: Oxford University Press, 761-789.

483 Martino G, Marks, LE. 1999. Perceptual and linguistic interactions in speeded classification: Tests 484 of the semantic coding hypothesis. Perception 28:903-924. DOI: $10.1068 / \mathrm{p} 2866$.

485 486 487 488

Martino G, \& Marks LE. 2001. Synesthesia: Strong and weak. Current Directions in Psychological Science 10:61-65. DOI: 10.1111/1467-8721.00116.

Miller GA, Johnson-Laird PN. 1976. Language and perception. Cambridge, MA: Belknap Press.

Ngo MK, Velasco C, Salgado A, Boehm E, O’Neill D, Spence C. 2013. Assessing crossmodal correspondences in exotic fruit juices: The case of shape and sound symbolism. Food Quality and Preference 28:361-369. DOI: 10.1016/j.foodqual.2012.10.004.

Noguchi K, Gel YR, Brunner E, Konietschke F. 2012. nparLD: An R Software Package for the nonparametric analysis of longitudinal data in factorial experiments. Journal of Statistical Software 50:1-23.

Osgood CE. 1960. The cross-cultural generality of visual-verbal synesthetic tendencies. Behavioral Science 5:146-169. DOI: 10.1002/bs.3830050204.

Osgood CE. 1964. Semantic differential technique in the comparative study of cultures. American Anthropologist 66:171-200. DOI: 10.1525/aa.1964.66.3.02a00880.

Osgood CE, Suci GJ, Tannenbaum PH. 1957. The measurement of meaning. Urbana, IL: University of Illinois Press. 
500 Palmer SE, Langlois TA, Schloss KB. 2015. Music-to-color associations of single-line piano

501 melodies in non-synesthetes. Multisensory Research 29:157-193. DOI: 10.1163/2213480850200002486.

503 Palmer SE, Schloss KB, Sammartino J. 2013. Visual aesthetics and human preference. Annual 504 Review of Psychology 64:77-107. DOI: 10.1146/annurev-psych-120710-100504.

505 Palmer SE, Schloss KB, Xu Z, Prado-León L.R. 2013. Music-color associations are mediated by 506 emotion. Proceedings of the National Academy of Sciences of the USA 110:8836-8841. DOI: 10.1073/pnas.1212562110.

Parise CV. 2015. Crossmodal correspondences: Standing issues and experimental guidelines. Multisensory Research 29:7-28. DOI: 10.1163/22134808-00002502.

Parise CV, Knorre K, Ernst MO. 2014. Natural auditory scene statistics shapes human spatial hearing. Proceedings of the National Academy of Sciences of the USA, 111:6104-6108. DOI:

Parise C, Spence C. 2013. Audiovisual cross-modal correspondences in the general population. In 10.1073/pnas.1322705111. Simner J, Hubbard E, eds., The Oxford handbook of synaesthesia. Oxford, UK: Oxford University Press, 790-815.

Piqueras-Fiszman B, Alcaide J, Roura E, Spence C. 2012. Is it the plate or is it the food? Assessing 517 the influence of the color (black or white) and shape of the plate on the perception of the food placed on it. Food Quality and Preference 24:205-208. DOI: 10.1016/j.foodqual.2011.08.011. Statistical Computing, Vienna, Austria. URL https://www.R-project.org/. 
521 Ramachandran VS, Hubbard EM. 2001. Synaesthesia--a window into perception, thought and 522 language. Journal of Consciousness Studies 8:3-34.

523 Salgado-Montejo A, Alvarado J, Velasco C, Salgado CJ, Hasse K, Spence C. 2015. The sweetest 524 thing: The influence of angularity, symmetry, and number of elements on shape-valence and 525 shape-taste matches. Frontiers in Psychology 6:1382. DOI: 10.3389/fpsyg.2015.01382.

526 Schifferstein HNJ, Tanudjaja I. 2004. Visualizing fragrances through colors: the mediating role of 527 emotions. Perception 33:1249-1266.

Spence C. 2011. Crossmodal correspondences: A tutorial review. Attention, Perception, \& 529 Psychophysics 73:971-995. DOI: 10.3758/s13414-010-0073-7.

Spence, C, Deroy O. 2014. Tasting shapes: a review of four hypotheses. Theoria et Historia 531 Scientiarum 10:207-238. DOI: 10.12775/ths-2013-0011.

Spence C, Ngo MK. 2012. Assessing the shape symbolism of the taste, flavour, and texture of 533 foods and beverages. Flavour 1:12. DOI: 10.1186/2044-7248-1-12.

Stein, B. E. (Ed.). (2012). The new handbook of multisensory processing. Cambridge, MA: MIT 535 Press.

Stewart PC, Goss E. 2013. Plate shape and colour interact to influence taste and quality judgments. Flavour 2:27. DOI: 10.1186/2044-7248-2-27.

Velasco C, Salgado-Montejo A, Marmolejo-Ramos F, Spence C. 2014. Predictive packaging 539 design: Tasting shapes, typographies, names, and sounds. Food Quality and Preference 34:88540 95. DOI: 10.1016/j.foodqual.2013.12.005. 
541 Velasco C, Woods A, Deroy O, Spence C. 2015a. Hedonic mediation of the crossmodal

542 correspondence between taste and shape. Food Quality and Preference 41:151-158. DOI:

$543 \quad$ 10.1016/j.foodqual.2014.11.010

544 Velasco C, Woods AT, Hyndman S, Spence C. 2015b. The taste of typeface. i-Perception 6:1-10.

$545 \quad$ DOI: $10.1177 / 2041669515593040$.

546 Velasco C, Woods AT, Liu J, Spence C. 2016. Assessing the role of taste intensity and hedonics

547 in taste-shape correspondences. Multisensory Research 29:209-221. DOI: 10.1163/22134808-

$548 \quad 00002489$

549 Walker P. 2012. Cross-sensory correspondences and cross talk between dimensions of connotative 550 meaning: Visual angularity is hard, high-pitched, and bright. Attention, Perception, \& 551 Psychophysics 74: 1792-1809. DOI: 10.3758/s13414-012-0341-9.

552 Walker L, Walker P. in press. Cross-sensory mapping of feature values in the size-brightness 553 correspondence can be more relative than absolute. Journal of Experimental Psychology: $554 \quad$ Human Perception and Performance.

555 Walker L, Walker P, Francis B. 2013. A common scheme for cross-sensory correspondences 556 across stimulus dimensions. Perception 41:1186-1192. DOI: 10.1068/p7149.

557 Wan X, Woods AT, van den Bosch J, McKenzie KJ, Velasco C, Spence C. 2014. Cross-cultural 558 differences in crossmodal correspondences between basic tastes and visual features. Frontiers 559 in Psychology 5:1365. DOI: 10.3389/fpsyg.2014.01365

560 Williams JM. 1976. Synaesthetic adjectives: A possible law of semantic change. Language 52: 561 461-478. DOI: $10.2307 / 412571$. 
562 Woods AT, Velasco C, Levitan CA, Wan X, Spence C. 2015. Conducting perception research over 563 the internet: A tutorial review. PeerJ 3:e1058. DOI: 10.7717/peerj.1058.

564 Yu N. 2003. Synesthetic metaphor: A cognitive perspective. Journal of Literary Semantics 32: 19565 34. DOI: $10.1515 /$ jlse. 2003.001. 
568 Figure 1. Shape stimuli used in Experiment 1.

569 Figure 2. Example of (A) a taste word and (B) a shape trial in Experiment 1.

570 Figure 3. Panel A presents the unrotated factor map of the polar scales in Experiment 1. Note that

571 only the label of the upper end of the scales is presented. Panel B presents the unrotated factor map

572 for the stimuli. The circles grouped the variables as a function of the two clusters identified in the

573 subsequent cluster analysis. Note that given that panel A and B show the unrotated visualizations,

574 the percentages for each component vary slightly from those presented in Table 1.

575 Figure 4. Dendrogram obtained by means of hierarchical cluster analysis in Experiment 1.

576 Figure 5. Mean ratings for each cluster and dimension in Experiment 1. The error bars represent

577 the standard error of the means.

578 Figure 6. Panel A presents the shape stimuli used in both tasks, Panel B a trial in the shape response 579 task, and Panel $\mathrm{C}$ a trial in the taste response task. Note that the shape stimuli are group in pairs as 580 used as responses for the shape response task.

581 Figure 7. Summary of the results of Experiment 2. Panel A presents accuracy and panel B presents 582 the mean reaction times (RTs) in both tasks as a function of congruence. The error bars represent 583 the standard error of the means. 


\section{Table 1 (on next page)}

Table 1

Varimax-rotated component matrix in Experiment 1 (see also Figure 4). 
1 Table 1. Varimax-rotated component matrix in Experiment 1 (see also Figure 4).

\begin{tabular}{ccc}
\hline \multirow{2}{*}{ Adjectives } & \multicolumn{2}{c}{ Component } \\
& 1 & 2 \\
\hline Passive - active & $\mathbf{. 9 9 5}$ & -.018 \\
Slow - fast & $\mathbf{. 9 8 6}$ & .033 \\
& & \\
Powerless - powerful & $\mathbf{. 9 8 6}$ & -.065 \\
& & \\
Weak - strong & $\mathbf{. 9 8 0}$ & -.125 \\
Shallow - deep & $\mathbf{. 9 3 4}$ & -.182 \\
Quiet - noisy & $\mathbf{. 9 3 3}$ & -.184 \\
Harsh - mild & $\mathbf{- . 8 2 5}$ & .565 \\
Sad - happy & .168 & $\mathbf{. 9 7 9}$ \\
Awful - nice & -.293 & $\mathbf{. 9 4 0}$ \\
Bad - good & -.311 & $\mathbf{. 9 1 9}$ \\
Light - heavy & .497 & $\mathbf{- . 8 2 5}$ \\
Dead - alive & .560 & $\mathbf{. 8 0 8}$ \\
Eigenvalues & 7.09 & 4.43 \\
\% of variance & $59.09 \%$ & $36.89 \%$ \\
\hline
\end{tabular}

2 


\section{Table 2 (on next page)}

Table 2

Hierarchical Cluster Analysis in Experiment 1. Note that the distance measure was rescale to $a \sim 0-1$ range, and that the largest jump occurs between steps four and five. The coefficient of the latter represents the distance between the cluster involving the round shapes and sweet taste word, and the cluster that includes the angular shapes and the other taste words. 
1 Table 2. Hierarchical Cluster Analysis in Experiment 1. Note that the distance measure was 2 rescale to $a \sim 0-1$ range, and that the largest jump occurs between steps four and five. The 3 coefficient of the latter represents the distance between the cluster involving the round shapes and 4 sweet taste word, and the cluster that includes the angular shapes and the other taste words.

\begin{tabular}{|c|c|c|c|c|c|c|}
\hline \multirow{2}{*}{ Stage } & \multicolumn{2}{|c|}{ Cluster combined } & \multirow{2}{*}{ Coefficients } & \multicolumn{2}{|c|}{ Stage cluster first appears } & \multirow{2}{*}{ Next stage } \\
\hline & Cluster 1 & Cluster 2 & & Cluster 1 & Cluster 2 & \\
\hline 1 & Sour & Bitter & .000 & 0 & 0 & 4 \\
\hline 2 & 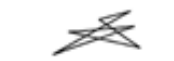 & $\sum_{3}^{2}$ & .010 & 0 & 0 & 6 \\
\hline 3 & ( ) & हु? & .031 & 0 & 0 & 5 \\
\hline 4 & Salty & Sour & .072 & 0 & 1 & 6 \\
\hline 5 & $\theta$ & Sweet & .220 & 3 & 0 & 7 \\
\hline 6 & $z$ & Salty & .400 & 2 & 4 & 7 \\
\hline 7 & $\approx$ & $\varnothing$ & 1.155 & 6 & 5 & 0 \\
\hline
\end{tabular}

5

6 


\section{Table 3 (on next page)}

Table 3

Experimental design used in Experiment 2. 
1 Table 3. Experimental design used in Experiment 2.

\begin{tabular}{|c|c|c|c|c|c|c|}
\hline Task & Congruence & $\begin{array}{c}\text { Instructions } \\
\text { (stimuli mapping) }\end{array}$ & Stimuli & Responses & $\begin{array}{l}\text { Unique } \\
\text { trials }\end{array}$ & Repetitions \\
\hline \multirow{2}{*}{$\begin{array}{l}\text { Shape } \\
\text { response }\end{array}$} & Congruent & $\begin{array}{l}\text { Sweet - round and } \\
\text { sour - angular }\end{array}$ & $\begin{array}{c}\text { Taste } \\
\text { words }\end{array}$ & $\begin{array}{l}\text { Angular or } \\
\text { round shape }\end{array}$ & 8 & \multirow{4}{*}{$\mathrm{X} 2$} \\
\hline & Incongruent & $\begin{array}{l}\text { Sweet - angular } \\
\text { and sour - round }\end{array}$ & $\begin{array}{l}\text { (sweet or } \\
\text { sour) }\end{array}$ & (four pairs x 2) & 8 & \\
\hline \multirow{2}{*}{$\begin{array}{l}\text { Taste } \\
\text { response }\end{array}$} & Congruent & $\begin{array}{l}\text { Round - sweet and } \\
\text { angular - sour }\end{array}$ & \multirow{2}{*}{$\begin{array}{l}\text { Shapes } \\
\text { (eight } \\
\text { shapes) }\end{array}$} & \multirow{2}{*}{ Sweet or sour } & 8 & \\
\hline & Incongruent & $\begin{array}{l}\text { Round - sour and } \\
\text { angular - sweet }\end{array}$ & & & 8 & \\
\hline
\end{tabular}

2

3 
1

Figure 1

Shape stimuli used in Experiment 1.

A

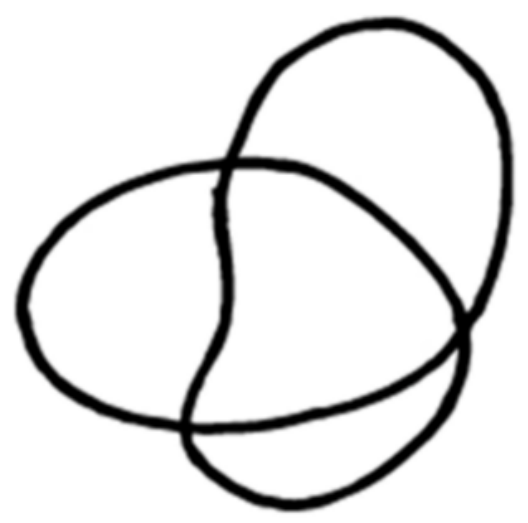

B

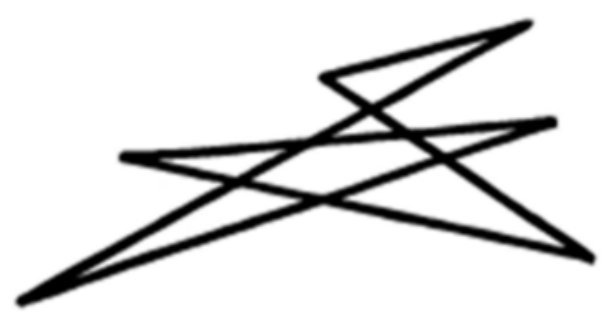

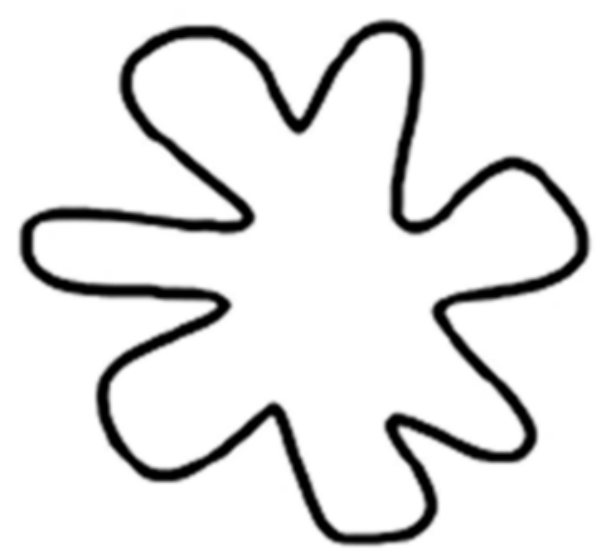

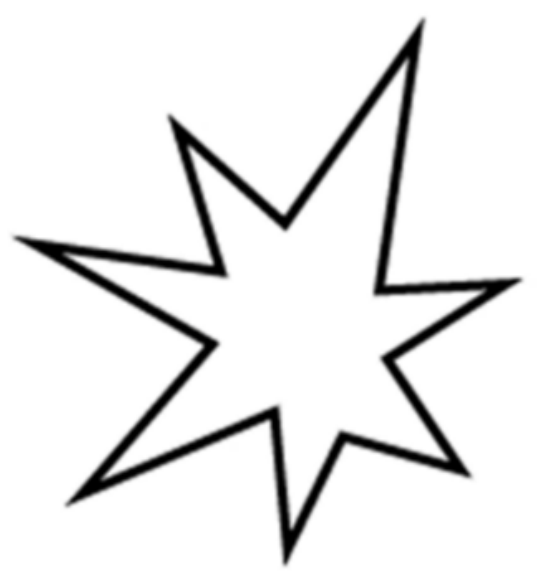


2

Figure 2

Example of (A) a taste word and (B) a shape trial in Experiment 1.

shallow




\section{3}

Figure 3

Panel A presents the unrotated factor map of the polar scales in Experiment 1. Note that only the label of the upper end of the scales is presented. Panel B presents the unrotated factor map for the stimuli. The circles grouped the variables as a function of the two clusters identified in the subsequent cluster analysis. Note that given that panel $A$ and $B$ show the unrotated visualizations, the percentages for each component vary slightly from those presented in Table 1. 


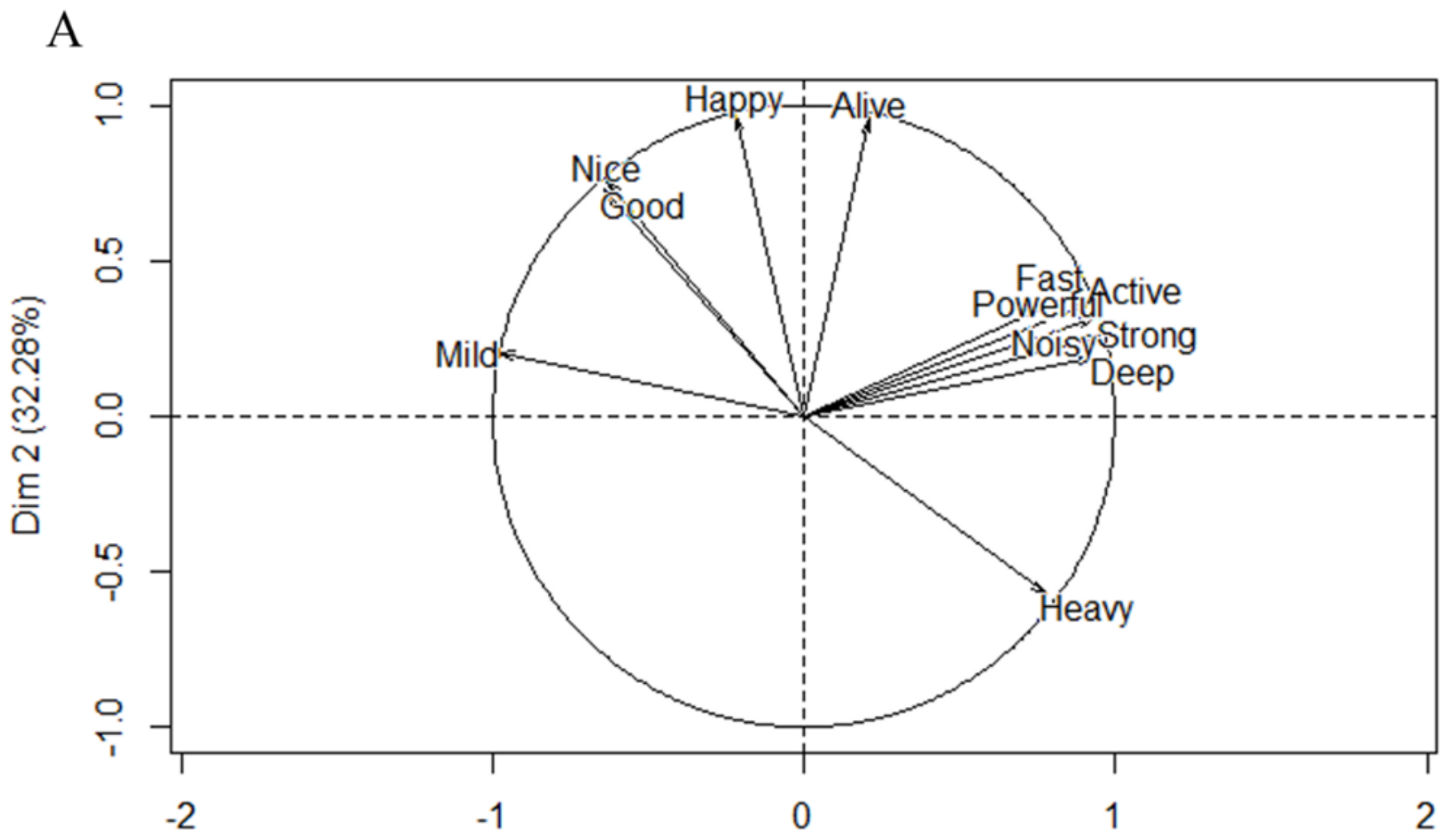

$\operatorname{Dim} 1(63.70 \%)$

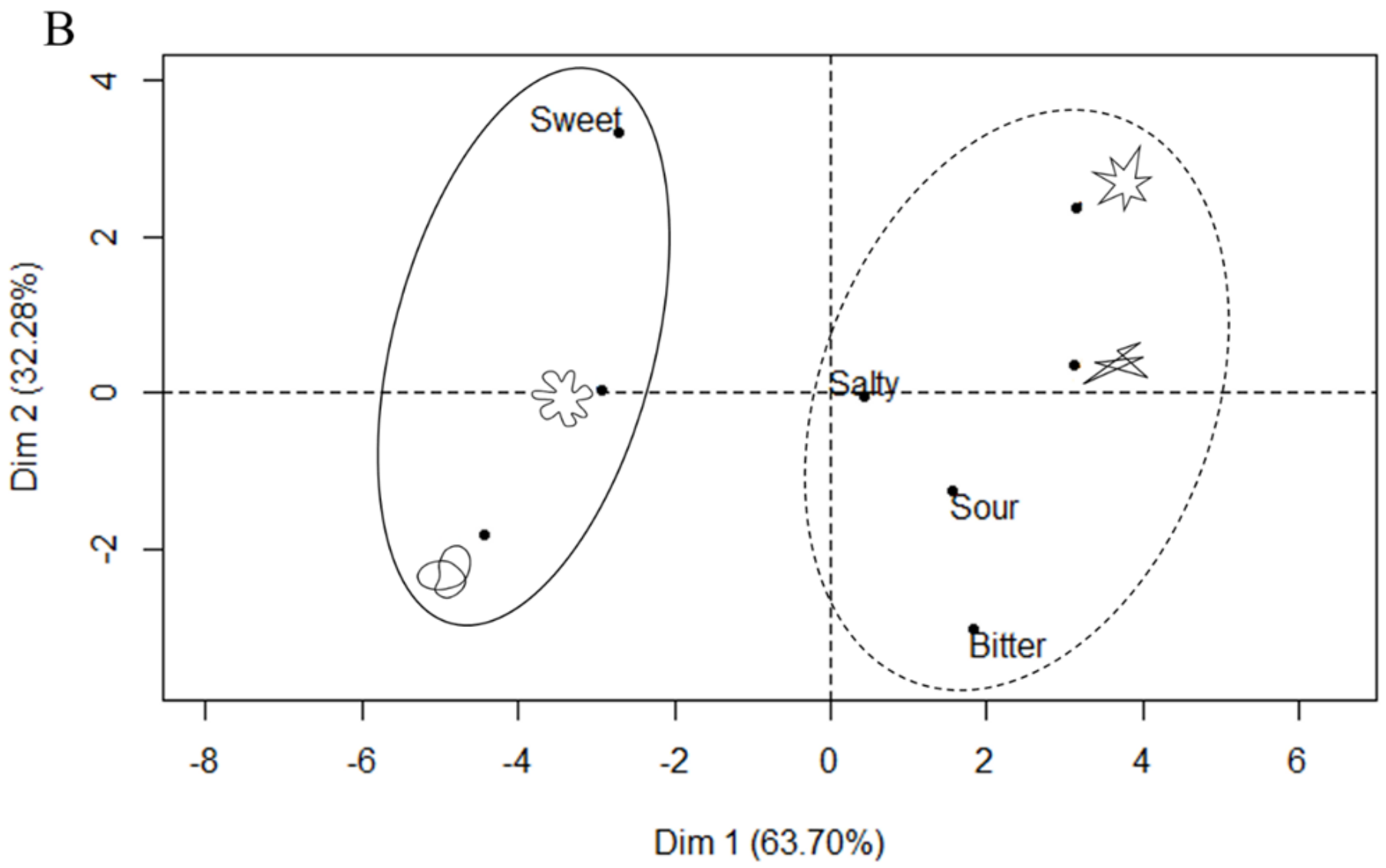


4

Figure 4

Dendrogram obtained by means of hierarchical cluster analysis in Experiment 1.

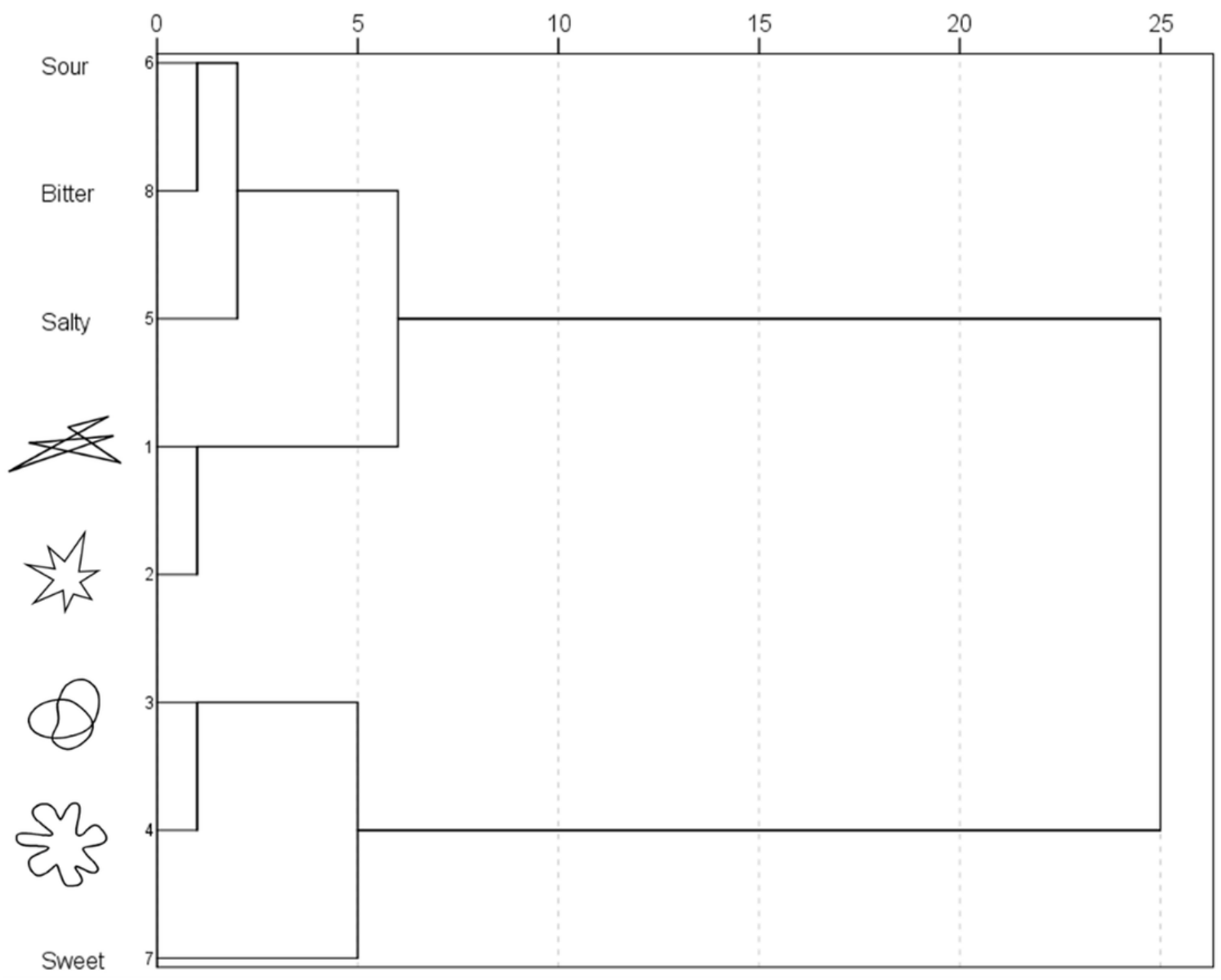




\section{5}

Figure 5

Mean ratings for each cluster and dimension in Experiment 1. The error bars represent the standard error of the means.

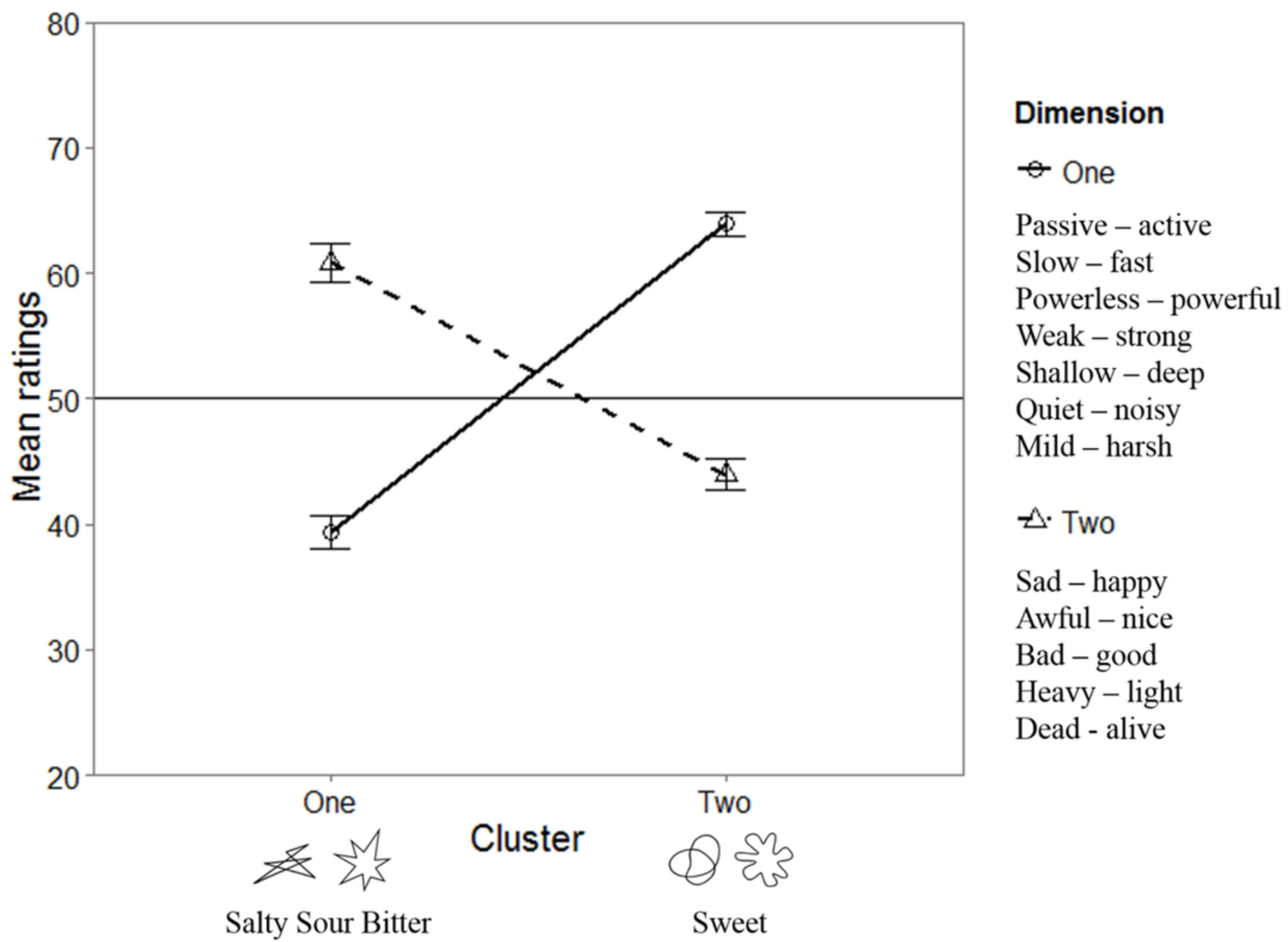


6

Figure 6

Panel A presents the shape stimuli used in both tasks, Panel B a trial in the shape response task, and Panel $\mathrm{C}$ a trial in the taste response task. Note that the shape stimuli are group in pairs as used as responses for the shape response task.

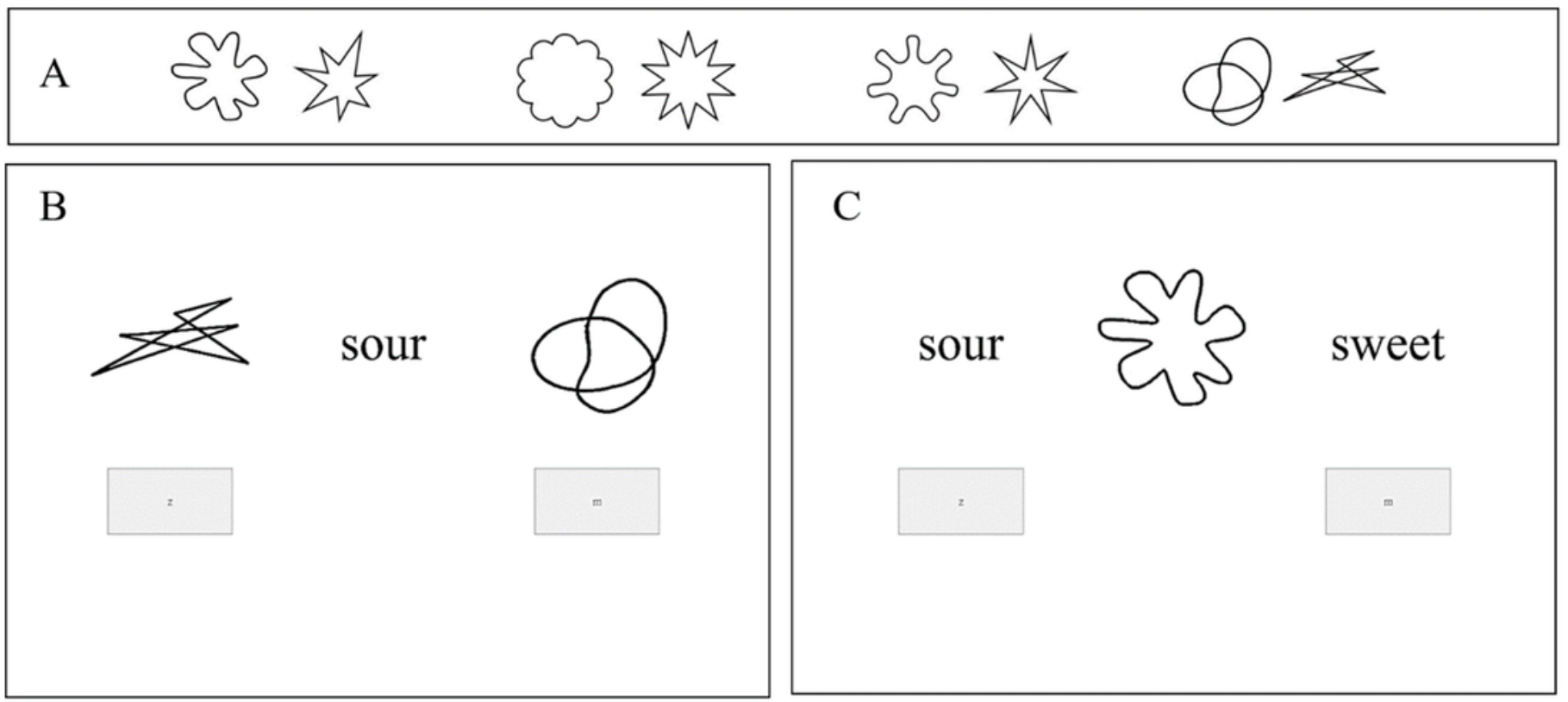


7

Figure 7

Summary of the results of Experiment 2. Panel A presents accuracy and panel B presents the mean reaction times (RTs) in both tasks as a function of congruence. The error bars represent the standard error of the means. 
A
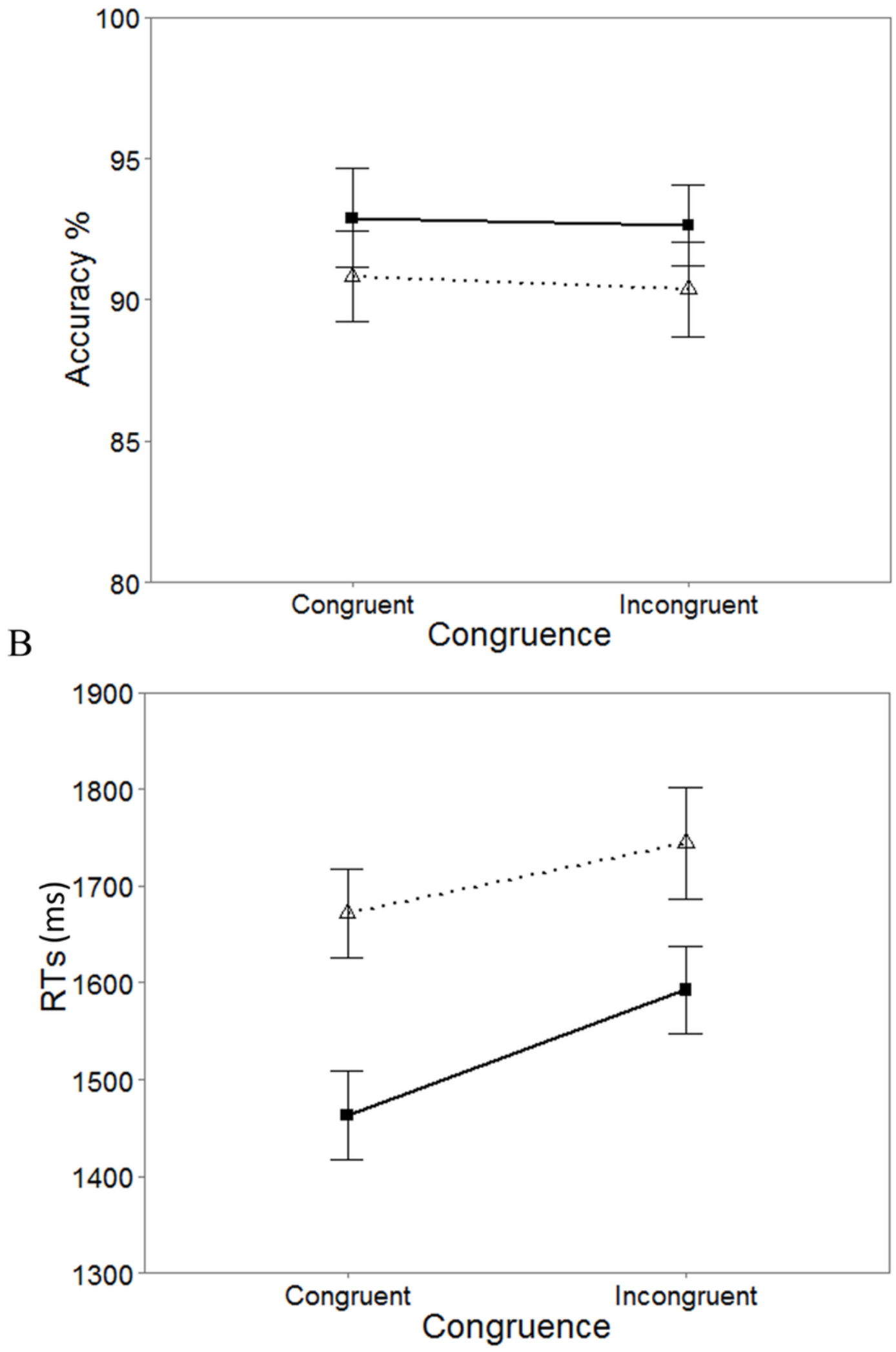

Task

- Respond with taste words -A. Respond with shapes 